\title{
Corela
}

Cognition, représentation, langage

11-1 | 2013

Vol. $11, \mathrm{n}^{\circ} 1$

\section{Modalisation dans le discours mathématique pédagogique écrit en anglais}

Eric Morvan

\section{OpenEdition}

\section{Journals}

Édition électronique

URL : http://journals.openedition.org/corela/2876

DOI : $10.4000 /$ corela. 2876

ISSN : 1638-573X

Éditeur

Cercle linguistique du Centre et de I'Ouest - CerLICO

Référence électronique

Eric Morvan, « Modalisation dans le discours mathématique pédagogique écrit en anglais », Corela [En ligne], 11-1 | 2013, mis en ligne le 20 juin 2013, consulté le 30 avril 2019. URL : http:// journals.openedition.org/corela/2876; DOI : 10.4000/corela.2876

Ce document a été généré automatiquement le 30 avril 2019

\section{cc)(1)(2)}

Corela - cognition, représentation, langage est mis à disposition selon les termes de la licence Creative Commons Attribution - Pas d'Utilisation Commerciale - Partage dans les Mêmes Conditions 4.0 International. 


\title{
Modalisation dans le discours
} mathématique pédagogique écrit en anglais

\author{
Eric Morvan
}

\section{Introduction}

1 Dans le cadre des théories énonciatives, on considère que tout discours ou texte est repéré par rapport à une situation d'énonciation qui comporte deux paramètres : le sujet énonciateur d'une part, le moment et le lieu de l'énonciation d'autre part. C'est uniquement grâce à ce repérage que le discours ou texte devient un énoncé. Modaliser consiste, pour le sujet énonciateur ${ }^{1}$, à prendre en charge un contenu propositionnel à partir d'une relation prédicative constituée, mais non encore repérée par rapport à une situation d'énonciation. Une définition possible est la suivante: la modalité est «l'expression de l'attitude du locuteur par rapport au contenu propositionnel de son énoncé » (Le Querler 1996: 61). Nous conviendrons en outre qu'il y a modalisation si l'énonciateur exprime un jugement ou une évaluation (Vold $2008: 57$ ).

Dans un article de recherche scientifique, le ou les auteurs ne peuvent se contenter d'exposer les résultats qu'ils ont obtenus et les interprétations qu'ils en donnent.

3 "The production of a research report such as an experimental research article depends critically 'on various processes of writing and reading which can be summarized as literary inscription. The function of literary inscription is the successful persuasion of readers [...]'. Underlying the use of persuasive language in scientific discourse, then, is the assumption that the information presented by the author may not be automatically accepted by the audience. » (Varttala $2001: 62$ )

Ainsi Vold (2008: 28) cite des travaux sur les stratégies argumentatives employées dans des articles scientifiques norvégiens de linguistique, d'histoire et de médecine. Elle constate que ces stratégies sont "ouvertes et explicites" pour les deux premières disciplines et "plus couvertes» en médecine; mais, "si ces stratégies sont moins 
aisément identifiables [en médecine] que les traces énonciatives dans les articles de linguistique et d'histoire, elles n'en sont pas moins présentes». En effet, comme le souligne Courtès (1991 : 250):

5 Le but de l'énonciation est moins de faire voir que de faire croire : même les énoncés les plus objectivés, tels ceux du discours scientifique, se veulent convaincants. Disons d'un mot que l'énonciateur manipule l'énonciataire pour que celui-ci adhère au discours qui lui est tenu.

6 Je me propose de justifier, dans le cadre du discours mathématique pédagogique, l'affirmation selon laquelle «la démarche de modalisation [est] absolument consubstantielle à la démarche scientifique» (Sionis 2002:2). Plus précisément, je me propose de montrer que l'utilisation de la modalisation par les auteurs peut s'expliquer par les stratégies que ceux-ci déploient afin d'atteindre les objectifs qu'ils ont assignés à leurs textes. Pour cela, j'examinerai quelques caractéristiques du discours mathématique en cherchant à mettre en évidence celles qui vont appeler une prise en charge explicite par l'énonciateur. Puis j'étudierai quelques points sur lesquels le discours mathématique pédagogique présente des spécificités comme les interprétations de shall et will, la forte présence de shall, les critères qui déterminent (dans certains cas) le choix entre can ou may, l'utilisation préférentielle de certains adverbes de modalité.

7 Pour le présent article, je me suis appuyé sur un corpus non numérisé constitué d'extraits de six ouvrages de mathématiques de niveau universitaire, à vocation pédagogique. Chaque extrait représente un passage continu d'une trentaine de pages en moyenne, dont l'emplacement dans l'ouvrage a été choisi aléatoirement. L'ensemble des extraits fait au total 181 pages. Quatre de ces ouvrages ont été écrits par des auteurs nord-américains et publiés aux Etats-Unis; les deux autres ont été écrits par des auteurs britanniques et publiés en Grande-Bretagne. Les modalisateurs que j'ai pris en compte sont les auxiliaires modaux, ainsi que certains adverbes de modalité et structures adjectivales extraposées. Dans mon corpus de travail, j'ai ainsi relevé 521 modalisateurs, dont 402 auxiliaires modaux (Morvan 2012 : 9).

\section{Eléments de caractérisation du discours mathématique pédagogique}

\subsection{Caractéristiques logiques et discursives}

8 Un article de recherche scientifique repose généralement sur des résultats expérimentaux. Le lecteur accepte a priori les données fournies par l'auteur même s'il ne connaît pas tous les détails du protocole des expériences. Mais les mathématiques ne sont pas une science expérimentale. Pour faire reconnaître la validité d'un résultat, l'auteur doit partir de prémisses qui sont des axiomes, des définitions ou des résultats précédemment obtenus, et il doit ensuite procéder par déductions successives en respectant les règles communément admises de la logique.

Une démonstration d'un théorème $(\mathrm{T})$ peut se définir comme un chemin qui, partant de propositions empruntées au tronc commun et de ce fait intelligibles par tous, conduit par étapes successives à une situation psychologique telle que (T) y apparaît comme évident. (René Thom, cité par Nordon 1999 : 12)

De ce fait, quand l'auteur arrive à des conclusions telles que : 
(1) This completes the proof. (C31) ${ }^{2}$

(2) Consequently, the equation $\mathrm{Ax}=\mathrm{b}$ has infinitely many solutions. (C322)

(3) By theorem 6.4, $W \approx \vec{E}^{n-m^{n-m}}$ and the result follows. (S140) celles-ci sont légitimées par les arguments développés précédemment.

Le terme " évident » est trompeur ; il ne signifie ni « facile », ni « immédiat ». L'évidence ne désigne pas quelque chose qui apparaît sans effort, mais elle est au contraire l'aboutissement d'un travail intellectuel. Pendant et après l'exposé d'une démonstration, le lecteur (ou l'auditeur) a besoin de temps pour comprendre. L'évidence est atteinte lorsqu'il n'a plus de question à poser et ne peut plus exprimer aucun doute. Parallèlement, après la fin de sa démonstration, l'auteur n'ajoute rien (ou l'orateur garde le silence).

11 Lorsqu'un mathématicien expose oralement une démonstration, le moment où il estime avoir atteint l'évidence se manifeste par son silence. [...] Ainsi les mathématiques sont un discours et ce discours a une étrange propriété : il convainc au moment où il se tait, parce que, parvenu à l'évidence, il ne sait plus quoi dire. (Nordon 1999 : 14)

Si le lecteur ou l'auditeur est psychologiquement prêt à accepter la conclusion qu'on lui propose, c'est qu'il a été convaincu par les explications qui lui ont été fournies. Pourtant, tout ne peut être détaillé dans une démonstration. Certaines connaissances nécessaires ont été exposées précédemment dans le même texte ou dans un autre ouvrage, et l'auteur y renvoie explicitement. Mais d'autres connaissances sont implicites : l'auteur suppose que le lecteur ou l'auditeur les possède et saura y faire appel pour compléter le raisonnement. Pour cette raison, certains enchaînements peuvent être dits " obvious ", " clear » ou « easy » et on trouvera des phrases telles que :

(4) Obviously, as regards the group-theoretic structure, a lattice of dimension $\mathrm{m}$ is a free abelian group of rank $\mathrm{m}$, so we can apply the terminology and theory of free abelian groups to lattices. (S135)

(5) Granted this, it is an easy matter to check that the two alternative factorizations in $\mathrm{Z}[\sqrt{ }-5]: 6=2.3=(1+\sqrt{ }-5)(1-\sqrt{ }-5)$ are just different groupings of the factors in the integers of $\mathrm{Q}(\sqrt{ }-5, \sqrt{2})$. (S172)

(6) The inclusion $a \subseteq B k \bigcap K$ is straightforward. (S167)

13 Faute de pouvoir présenter toutes les étapes de son raisonnement, l'auteur doit faire accepter au lecteur que ce qui n'a pas été explicité n'avait pas besoin de l'être. Dans ce cas, il y a une forte prise en charge de l'énoncé par le sujet énonciateur qui évalue ou commente ses assertions. On constate donc une modalisation de l'énoncé, qui peut se manifester par la présence de plusieurs marqueurs d'interprétations différentes dans une même phrase. Ces marqueurs peuvent servir à exprimer une prédiction, une nécessité logique (\$1.3), une possibilité, une capacité (\$2.1), un renforcement de l'assertion, une appréciation (\$3.1), etc. Voici quelques exemples :

(7) It would be nice, of course, if $\Psi(t)$ were the product of $f(t)$ and $y 2(t) / a$, but this is obviously false. However, there is an extremly interesting way of combining two functions $\mathrm{f}$ and $\mathrm{g}$ together to form a new fonction $\mathrm{f}^{*} \mathrm{~g}$, which resembles multiplication, and fot which $L\{f * g)(t)\}-L\{f(t)\} \times L\{g(t)\}$ (B252)

(8) We shall ordinarily use an indeterminant in describing polynomials, but there will be times, especially when multiplying or dividing, when it is useful to recall that a polynomial may be thought of as a tuple of coefficients, and "detach the coefficients". (C127)

(9) Since digital computer evaluates a function for an irrational argument about as easily as for a rational one, the Gaussian method is attaining the popularity it deserves, and should be used when feasible. In general, it should not be used if the integrand is available only in the form of equally spaced tabular points, for this would require some interpolation formula in 
the case of the irrational abscissas, so we might just as well use directly the Newton-Cotes formula of the same accuracy as the interpolation formula that would have been used. (G94-95)

\subsection{Caractéristiques fonctionnelles}

14 Un texte mathématique se présente globalement comme une succession de définitions et de résultats ${ }^{3}$, chaque résultat étant justifié par un enchaînement de raisonnements et de calculs. A ces constituants obligatoires s'ajoutent, dans les textes à visée pédagogique, des éléments facultatifs qui sont les remarques, les commentaires, les exemples et les exercices. Suivant la distinction établie par Petit (1993: 239), en mettant de côté les définitions et les résultats, on peut identifier deux fonctions différentes du discours mathématique.

En premier lieu, il y a la fonction intra-démonstrative qui apparait dans les parties où un raisonnement ou un calcul est développé pour démontrer un résultat (énoncé antérieurement ou postérieurement). Ces parties sont parfois précédées du titre explicite de Proof ou Solution. Les énoncés sont le plus souvent non modalisés, en particulier lorsqu'ils accompagnent des calculs détaillés. Certains passages présentent par contre des énoncés modalisés, lorsque l'auteur veut convaincre et guider le lecteur sans pour autant expliciter tous ses raisonnements et calculs. Exemples :

(10) Since we have three constants at our disposal, we can expect our formula to be exact for polynomials up through degree two. In particular, it must be exact for the cases $\mathrm{y}=1, \mathrm{y}=\mathrm{x}-\mathrm{x} 0, \mathrm{y}=(\mathrm{x}-\mathrm{x} 0) 2$. $(\mathrm{G} 85)$

(11) A polynominal $p(z)$ in $\hat{E}[z]$ may be written as

$\mathrm{p}(\mathrm{z})-\mathrm{p}(\mathrm{x}+\mathrm{iy})-\mathrm{p}_{1}(\mathrm{x}, \mathrm{y})+\mathrm{i} \mathrm{p}_{2}(\mathrm{x}, \mathrm{y})$

where $\mathrm{p}_{1}(\mathrm{x}, \mathrm{y})$ and $\mathrm{p}_{2}(\mathrm{x}, \mathrm{y})$ are real polynomials in the real variables $\mathrm{x}, \mathrm{y}$.

Then $|\mathrm{p}(\mathrm{z})|$ may be written as $|p(z)|-\sqrt{p_{1}(x, y)^{2}+p_{2}(x, y)^{2}}$. (C138)

(12) Hence $\mathrm{p}^{2}+\mathrm{pq}-\mathrm{q}^{2}-0$ or $(2 \mathrm{p}+\mathrm{q})^{2}-5 \mathrm{q}^{2}$, which is plainly impossible. (H164)

En second lieu, le discours mathématique a une fonction métadiscursive : celle-ci apparaît dans les parties où l'auteur annonce, commente ou complète les raisonnements, les calculs, les définitions ou les résultats. Par exemple les énoncés modalisés (7) à (9) relèvent de cette fonction. En reprenant la classification utilisée dans la théorie des opérations énonciatives ${ }^{4}$, nous pouvons identifier :

- des modalités assertives (type 1 ) :

(13) The conclusion is not confined to quadratic fields, but it is only in such fields that we have defined Ny and are in a position to state it precisely. (H212)

- des modalités épistémiques (type 2) :

(14) Simpson's rule is probably the most widely used quadrature formula. (G81)

- des modalités appréciatives (type 3):

(15) It is interesting that while there is a procedure for finding roots of any polynomial of degree 4 analogous to that of degree 3 , there is no corresponding way to find the roots of a polynomial of degree $\left[\begin{array}{rl}20 \\ 0\end{array} 5\right.$. (C138)

- des modalités radicales (type 4):

(16) We can regard our problem from two different points of view. (H155)

Selon le style propre à chaque auteur, on peut, lors d'une démonstration, avoir un texte à fonction uniquement intra-démonstrative (figure 1), ou bien un texte où les deux fonctions, intra-démonstrative et métadiscursive, sont développées simultanément (figure 2). Dans le premier cas, les remarques et commentaires sont souvent regroupés 
après la démonstration sous les titres Remark ou Note ; dans le deuxième cas, ils sont insérés dans le cours de la démonstration.

18 Les exemples ou contre-exemples constituent en eux-mêmes des commentaires de résultats; ils relèvent donc de la fonction métadiscursive. Mais à l'intérieur de l'exposition d'un exemple, il peut y avoir des calculs -qui vont donc relever de la fonction intra-démonstrative.

Figure 1 : Stewart \& Tall. Algebraic Number Theory.

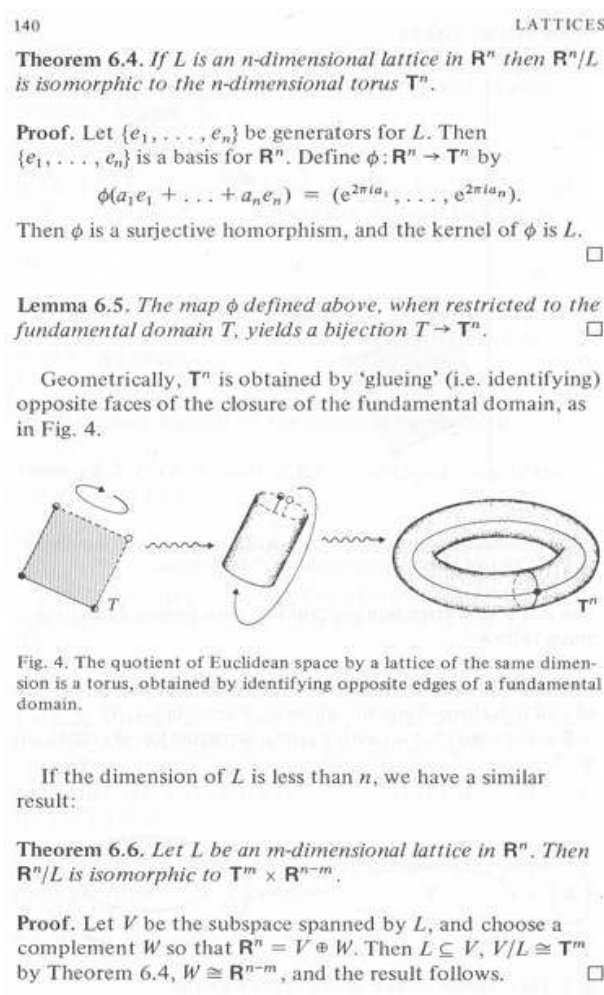


Figure 2 : Grove. Brief Numerical Methods.

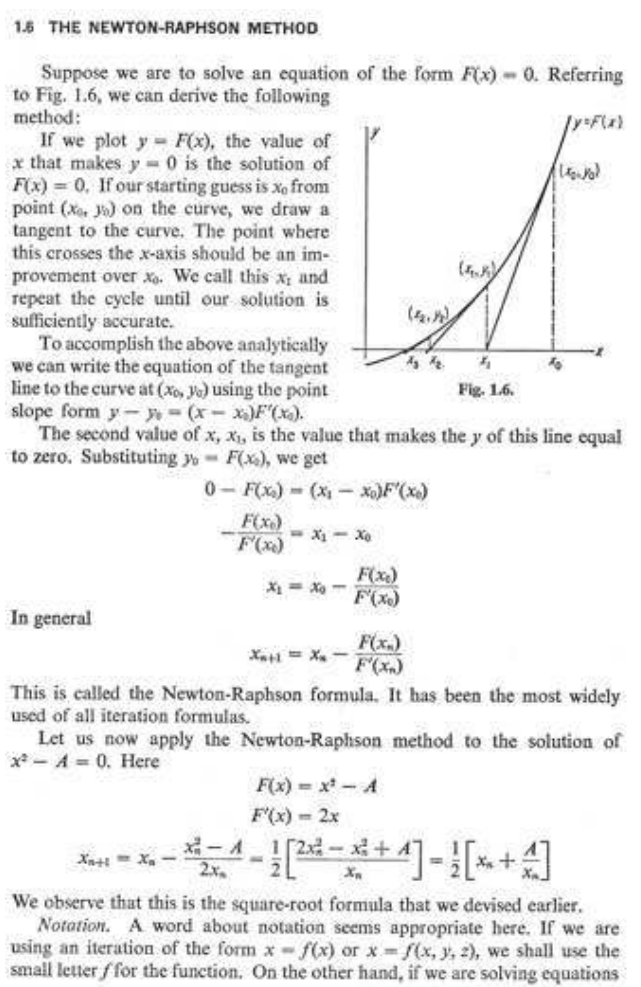

\subsection{Classification et répartition des valeurs de shall et will}

Dans mon corpus de travail, la quasi-totalité des 53 occurrences de shall et la grande majorité ${ }^{5}$ des 56 occurrences de will appartiennent à des parties de texte à fonction métadiscursive. Dans presque tous les exemples relevés, shall et will ont une fonction de renvoi à l'avenir

- soit avec une valeur purement temporelle :

(17) We use partial fractions to implement the idea that while it is hard to find the coefficients of $x n$ in a product of power series, it is easy in a sum of power series. So we shall use partial fractions on the right side of (1) to get a nice expression for a (n). (C153)

(18) In this section we will first present a physical justification of the method of Dirac. Then we will illustrate how to solve the initial-value problem (1) by the method of Laplace transforms. Finally we will indicate very briefly the "germ" of Laurent Schwartz's brilliant idea. (B245)

- soit avec une valeur " temporo-argumentative ", où « l'effet de futur s'accompagne d'un rôle fonctionnel dans l'argumentation" (Petit 1993: 256). L'énonciateur pourrait se contenter d'une assertion simple. Il choisit pourtant de reporter à un moment ultérieur la validation de la relation prédicative. C'est le cas par exemple lorsque l'auteur pose les notations ou les définitions qu'il va utiliser dans une démonstration à venir : "will [ou shall] permet [...] de procéder à la mise en place d'une convention proposée en attente de validation » (Petit 1993 : 257). Exemples :

(19) We shall retain the symbols $\langle\alpha>,\langle\alpha, \beta>$ to denote the ideals in $D$ generated by $\alpha$ and by $\alpha, \beta \varepsilon D$. (S167)

(20) A homomorphism $f: G \rightarrow G^{\prime}$ which establishes an isomorphism between $G$ and its image in $G$ ' will also be called an embedding. (L12) 
certains énoncés qui appartiennent à une partie de texte à fonction intradémonstrative. Ils font intervenir deux propositions $P$ et $Q$ dans une structure if $Q$ then $P$ ou if $Q, P^{6}$, et will apparaît dans la proposition P. Nous dirons avec Petit (1993:257) que will a ici une valeur « logico-déductive ». Exemples :

(21) If I0 is not very small, then $f(t)$ will be quite large in the interval $t_{0} \leq t \leq t_{1}$. (B244)

$\mathrm{Q}: \mathrm{I0}$ is not very small $\Rightarrow \mathrm{P}: \mathrm{f}(\mathrm{t})$ is quit large in the interval $\mathrm{t}_{0} \leq \mathrm{t} \leq \mathrm{t}_{1}$

(22) If there exists a proper subgroup $H$ of $G$ whose index is prime to $p$. then a p-Sylow subgroup of $H$ will also be one of $G$, and our assertion follows by induction. (L23)

$Q$ : there exists a proper subgroup $H$ of $G$ whose index is prime to $p=P$ : a $p$-Sylow subgroup of $H$ is also one of $G$

Signalons que must, lorsqu'il a une interprétation radicale de nécessité, apparaît lui aussi souvent dans une proposition $\mathrm{P}$ qui est la conséquence d'une proposition $\mathrm{Q}^{7}$ :

(23) Since $\mathrm{K}$ is a field this means that either $\delta$ is identically zero or $\delta$ is injective. But

$\delta(1)-(1,1, \ldots, 1) \neq 0$ so $\delta$ must be injective. (S154)

$\mathrm{Q}: \delta(1)-(1,1, \ldots, 1) \neq 0 \Rightarrow \mathrm{P}: \delta$ is injective.

Dans ce cas, must peut être remplacé par will :

(24) However, as with polynomials, it can be proved that if $F$ is an infinite field, then two rational functions which have the same values when evaluated on infinitely many elements of F must be equal. (C145)

(24') However, as with polynomials, it can be proved that if $F$ is an infinite field, then two rational functions which have the same values when evaluated on infinitely many elements of $F$ will be equal.

L'utilisation de must dans (24) a pour effet de mettre l'accent sur la nécessité logique de valider la relation prédicative <many elements of $F /$ be equal $>$, alors que l'utilisation de will dans (24') marque une prédiction de validation. Toutefois, will ayant une valeur logicodéductive, cette prédiction est présentée comme certaine.

\section{Modalités dominantes dans le discours mathématique pédagogique}

\subsection{Quelques facteurs favorisant ou limitant la modalisation}

Il est possible de mettre en évidence des circonstances qui déclenchent la modalisation d'un énoncé mathématique. Dans les exemples (25) et (26), il s'agit d'une modalité radicale, où can a une interprétation de possibilité ou de capacité (Gilbert 2001 : 43, 47).

(25) We know also that an algebraic number, which is an irrational of a comparatively simple type, cannot be 'too rapidly' approximable, while the transcendental numbers of Theorem 192 have approximations of abnormal rapidity. (H163)

Dans la première partie de cette phrase, l'auteur suppose connue par le lecteur une propriété des nombres algébriques, à savoir qu'un tel nombre ne peut pas être approximé "trop rapidement»; comme il ne donne pas dans ce passage de preuve explicite, l'énonciateur représenté par l'auteur prend en charge l'affirmation en utilisant cannot. $A$ contrario, dans la deuxième partie, il n'y a pas nécessité d'une modalisation car la propriété énoncée a été explicitement prouvée quelques lignes auparavant ; l'auteur n'a nul besoin de convaincre son lecteur qui peut se reporter au théorème 192 en cas de doute.

(26) The first assertion follows from the second, since if $G$ has center $\mathrm{Z}$ and we have an abelian tower for $\mathrm{G} / \mathrm{Z}$ by induction, we can lift this abelian tower to $\mathrm{G}$ to show 
that $G$ is solvable. To prove the second assertion, we use the class equation (G:1)$\operatorname{card}(\mathrm{Z})+\Sigma\left(G: G_{x}\right)$, the sum being taken over certain $x$ for which $\left(G: G_{x}\right) \neq 1$. Then $p$ divides ( $\mathrm{G}: 1)$ and also divides every term in the sum, so that $\mathrm{p}$ divides the order of the center, as was to be shown. (L25) présentant des modalités appréciatives ou radicales. Dans le discours scientifique non mathématique, la modalité épistémique est également rencontrée; elle découle à la fois d'une incertitude réelle de l'énonciateur et d'une atténuation par précaution de ses affirmations (phénomène de hedging) (Vold 2008: 13-14). Dans le cadre d'un article de recherche, de nombreuses raisons peuvent expliquer cette atténuation: imprécision des résultats expérimentaux, possibilité d'une remise en question du choix du cadre théorique ou tout simplement exposition de conjectures (Varttala 2001: 67-69). Et l'utilisation de modalisateurs épistémiques est un moyen privilégié d'expression de l'atténuation: "hedging has characteristically been linked to epistemic modality, because the meaning of both epistemically modal devices and hedges is closely related to the sender's degree of confidence regarding what is being said» (Varttala 2001 : 27). En revanche, dans le discours mathématique, l'auteur n'a généralement aucune incertitude sur les points de départ de son raisonnement, ni sur la validité des enchaînements logiques, donc aucune raison d'atténuer ses propos. Par conséquent la présence de modalité épistémique semble exclue, en tout cas dans les parties de texte à fonction intra-démonstrative. Effectivement, sur les 521 marqueurs modaux relevés dans mon corpus de travail, je n'en ai guère trouvé qu'une quinzaine qui aient une interprétation clairement épistémique (Morvan 2012 : 28), et presque tous sont inclus dans des parties à fonction métadiscursive, comme par exemple :

(27) These rough considerations suggest that, if we compare the $a_{n<} \Phi(n)$, and

$$
\begin{aligned}
& \left|\frac{p}{q}-\xi\right|<\frac{1}{q \chi(q)} \text { there sould be a certa } \\
& \qquad \sum \frac{1}{\phi(n)}, \sum \frac{1}{\chi(q)}{ }_{(\mathrm{H} 169)}
\end{aligned}
$$

L'exemple qui suit est une exception: il est inclus dans une partie à fonction intradémonstrative. May y est utilisé car l'auteur ne peut pas, sans étude supplémentaire, décider s'il existe ou non des fonctions rationnelles ayant la propriété mentionnée.

(28) There may exist rational functions in $F(x)$ which cannot be defined as functions on $F$ at all. (C144)

\subsection{Forte présence de shall}

Le discours mathématique se veut objectif et dépersonnalisé. Il a pour ambition d'établir des vérités universelles et permanentes, qui sont les mêmes quelle que soit l'identité de celui qui les énonce. Donc, bien que tout ouvrage mathématique ait un auteur et soit destiné à des lecteurs, l'identité de l'un et des autres n'est pas importante. En conséquence les énoncés sont généralement pris en charge par un sujet énonciateur indéterminé, voire effacé : «les caractéristiques du discours mathématique, qui tiennent 
notamment [...] à la "discursivité explicitement réglée qui caractérise le processus discursif scientifique" entraînent [...] un "effacement du sujet de l'énonciation"8 " (Petit $1993: 8)$.

L'établissement de résultats incontestables étant l'objet principal des textes mathématiques, on remarque que ce but est mieux servi par l'utilisation de shall que par celle de will. En effet une prédiction effectuée dans un énoncé utilisant shall a un caractère de certitude beaucoup plus affirmé que dans un énoncé utilisant will. Avec shall, l'énonciateur fait une prédiction qui a pour caractéristique d'être inéluctable car «l'énonciateur dit que le sujet de la relation prédicative est lié par la prédiction de

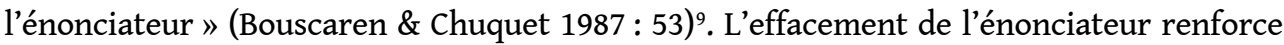
en outre l'inéluctabilité de la prédiction : « [le] haut degré de certitude peut à notre avis ${ }^{10}$ être vu, non comme un fort engagement de l'énonciateur [...] mais comme une mise en retrait de ce dernier, débarrassant sa prise en charge de toute espèce de subjectivité, et lui donnant par là-même une forme d'objectivité difficilement contestable » (Gilbert $2001: 89$ ).

30 J'ai dénombré les occurrences des syntagmes we shall/we will dans mon corpus et j'ai constaté que shall apparaît trois fois plus souvent que will ${ }^{11}$, y compris dans les quatre ouvrages nord-américains. Il semble donc que ne s'appliquent pas au discours mathématique pédagogique les deux constatations bien connues selon lesquelles l'usage de shall est en constante régression et limité à l'anglais britannique. Toutefois les raisons invoquées ci-dessus pour expliquer cet emploi préférentiel de shall ne sont pas toujours opérantes. En témoignent les deux énoncés suivants où les calculs entrepris à la suite de chaque énoncé sont très semblables :

(29) We shall derive the trapezoidal rule. (G84)

(30) We will derive the predictor. (G103)

31 On peut toutefois remarquer que, après (29), l'auteur cherche à retrouver une formule donnée auparavant; donc son calcul s'accompagne de l'obligation d'obtenir un résultat déjà connu. Au contraire, après (30), l'auteur cherche à établir une nouvelle formule ; son calcul n'est pas soumis à une contrainte aussi forte et il reste une incertitude : la formule cherchée peut se révéler par exemple inutilisable en pratique ${ }^{12}$.

\subsection{Choix entre can et may pour l'expression d'une hypothèse ou d'un résultat}

Can et may sont les deux auxiliaires modaux les plus fréquents dans mon corpus de travail : 131 occurrences pour can (dont 15 pour cannot) et 77 occurrences pour may (Morvan 2012 : 37-40). Ils ont toujours (pour can) ou presque toujours (pour may) une interprétation radicale de possibilité, paraphrasable par « it is possible for ... to ... » (Gilbert 1987 : 89, 95). Dans cette situation, can et may sont souvent mutuellement substituables. On peut toutefois mettre en évidence deux différences énonciatives et sémantiques induites par l'usage d'un modal plutôt que de l'autre.

Une première différence peut être expliquée en comparant ces deux énoncés, empruntés à Gilbert (1987 : 253) :

We operate what may be described as a gigantic tutorial system.

We operate what can be described as a gigantic tutorial system. 
« Dans le premier énoncé, may marque clairement qu'il y a d'autres descriptions possibles et peut-être préférables à celle qui est proposée, alors que can, dans le deuxième énoncé, ne fait qu'indiquer que la description avancée appartient au domaine du possible et ne laisse rien entendre quant à d'éventuelles autres descriptions » (Gilbert 1987 : 253). Même si may n'a pas ici une interprétation épistémique, l'énonciateur, en l'utilisant, continue à envisager à la fois la validation et la non-validation de la relation prédicative. Il y a prise en compte de l'altérité ${ }^{13}$.

Comparons maintenant les deux énoncés suivants, empruntés à Lapaire \& Rotgé (1998: 502) :

Driving can be dangerous : en soi, conduire peut être dangereux.

Driving may be dangerous : au vu des circonstances, conduire peut être dangereux.

On constate ici une deuxième différence entre can et may: «can fait référence [à des] capacités théoriques [...] alors que may est lui plutôt utilisé pour renvoyer à des manifestations pratiques, concrètes des capacités en question.» (Gilbert $2001: 60-61) . \mathrm{Ou}$ encore : «avec may [...] on insisterait plutôt sur les manifestations quantitatives de la propriété en question, sur le fait qu'il existe des occurrences de la relation prédicative envisagée ", alors que, avec can, on met en avant la "propriété constitutive de ces occurrences » (Gilbert $2001: 62$ ).

Une particularité de mon corpus est la présence d'énoncés du type we may+ $\mathrm{V}^{14}$ et l'absence totale d'énoncés du type we can+V, où $\mathrm{V}$ est un verbe posant une hypothèse : assume ou suppose.

(31) Since the time interval is extremely small, we may assume that the position of the particle does not change while the force $f(t)$ acts. (B245)

(32) Now $2=12+12+02+02$ so we may suppose $p$ is odd. (S149)

Symétriquement, j'ai remarqué la présence d'énoncés du type we $c a n+\mathrm{V}$, one can $+\mathrm{V}$, can be $+\mathrm{V}$-ed et it can be $+\mathrm{V}$-ed+that, où $\mathrm{V}$ est un verbe marquant la fin d'un raisonnement ou d'un calcul, et la quasi-absence d'énoncés analogues avec may ${ }^{15}$. Les verbes rencontrés sont find , obtain, prove et show.

(33) We can find a greatest common divisor of two polynomials by using the division theorem many times. (C132)

(34) It can be shown that this addition defines a monoid structure on $M$, whose unit element is the class of the ordinary 2-sphere. (L8)

(35) Gauss posed the question of whether a numerical integration formula can be obtained which gives an exact result for polynomials of degree greater than $n$. (G88)

Ces deux constatations peuvent s'expliquer par la prise en compte de l'altérité par may et sa non-prise en compte par can. En effet :

- une hypothèse est un choix entre plusieurs possibilités, fait au début d'un raisonnement. A ce stade, l'énonciateur garde présentes à l'esprit les autres hypothèses qu'il aurait pu faire. D'où l'utilisation de may plutôt que can.

- un résultat est le point final d'un raisonnement ; il peut ou non avoir été annoncé à l'avance, mais, dans les deux cas, l'énonciateur a prévu d'arriver précisément à ce résultat et n'est intéressé par aucun autre. D'où l'utilisation de can plutôt que may.

La deuxième différence entre can et may rappelée ci-dessus permet d'expliquer l'énoncé suivant :

(36) Now, we may assume that $a>0$ in Equation (1), for otherwise we can multiply both sides of the equation by -1 . (B245) 
le cas où $a<0$, il est possible de multiplier les deux termes de l'équation par -1 , et ainsi de changer le signe de $a$. On peut donc se contenter de traiter le cas où $a>0$. Mais, du fait de cette hypothèse, la multiplication par -1 reste une action potentielle et, en utilisant can, l'énonciateur signale que la possibilité énoncée reste théorique. Par contre, may introduit une hypothèse qui va effectivement être adoptée pour la suite des calculs comme le montre le début de la phrase suivante : In this case (see Section 2.6), [...].

Les deux seuls exemples d'utilisation avec may d'un verbe exprimant un résultat sont:

(37) The error via Simpson's rule may be found in a similar fashion. (G86)

(38) It may be proved by the methods of this section that $\alpha_{1} \mathrm{e}^{\beta_{1}}+\alpha_{2} \mathrm{e}^{\beta 2}+\ldots+\alpha_{\mathrm{x}} \mathrm{e}^{\beta \mathrm{x}} \approx 0$. (H176)

En se référant au contexte, on constate que, dans (37) et (38), le résultat que donne l'auteur n'est pas prouvé ; l'utilisation de may semble indiquer qu'il juge la démonstration non essentielle. En revanche, dans les 20 exemples où can est utilisé, le résultat énoncé est explicitement démontré 13 fois et non démontré 5 fois (Morvan 2012 : 46) ${ }^{16}$.

Pour finir, considérons les trois énoncés suivants, extraits du même ouvrage :

(39) The field $F$ is contained in $F[x]$ : an element a of $F$ can be thought of as a polynomial of degree 0 , namely, $a+0 x+0 \times 2+\ldots$. (C126)

(40) We shall ordinarily use an indeterminant in describing polynomials, but there will be times, especially when multiplying or dividing, when it is useful to recall that a polynomial may be thought of as a tuple of coefficients, and "detach the coefficients". (C127)

(41) Similarly, polynomials in three variables may be thought of as polynomials in one variable with coefficients in $F[x][y]$, etc. (C128)

Interrogé par mèl sur les raisons de l'utilisation de can be thought of dans (39) et de may be thought of dans (40) et (41), l'auteur L. Childs a d'abord répondu : " My first response to your question about my uses of "may be thought of" vs. "can be thought of" is that I never explicitly thought about the use of one or the other -in the places you quoted, I viewed them as interchangeable ». Puis, après réflexion :

- dans (40), «there is an implied permission (and in fact an encouragement) to the reader to add or multiply two polynomials by detaching the coefficients »;

- (41) « is more problematical. I changed my mind about this one overnight. What I implicitly may have had in mind is that viewing $\mathrm{F}[\mathrm{x}, \mathrm{y}, \mathrm{z}]$ as polynomials in $\mathrm{z}$ with coefficients that are polynomials in $\mathrm{x}$ and $\mathrm{y}$ is the best way to view $\mathrm{F}[\mathrm{x}, \mathrm{y}, \mathrm{z}]$. For example, the usual proof that $\mathrm{F}$ $[\mathrm{x}, \mathrm{y}, \mathrm{z}]$ has unique factorization requires that point of view ";

- " [therefore] the linguistic distinction I would make between [example (39)] and the other two is the implicit encouragement noted above $»^{17}$.

L'utilisation de may de préférence à can dans (40) et (41) semble être une conséquence de la prise en compte de l'altérité par may. En effet, dans (39), il n'y a effectivement pas d'alternative au choix de considérer un élément de $\mathrm{F}$ comme un polynôme de degré 0 , d'où l'utilisation de can. Par contre, dans (41), comme L. Childs l'explique lui-même, il y a d'autres choix possibles ${ }^{18}$, ce que marque l'utilisation de may. Or l'existence de plusieurs choix implique la nécessité pour l'auteur d'encourager le lecteur à faire le même choix que lui afin que l'exposé mathématique puisse être poursuivi. Commet Gresset (2001:202) le dit à propos d'un énoncé fort semblable à (39), (40) et (41), « la valeur de base de may, la prise en compte de l'altérité, [...] revient, dans les contextes intersubjectifs, au coénonciateur, qui se voit attribuer ici un rôle essentiel, et c'est la différence fondamentale avec can ». 


\section{Marqueurs de modalité appréciative}

\subsection{Adverbes de modalité}

Les adverbes de modalité sont définis par Guimier (1988:253) comme «l'ensemble des adverbes qui définissent la valeur de vérité de l'énoncé ». En utilisant un adverbe de modalité, l'énonciateur apporte une information sur les fondements du jugement qu'il porte et il indique avec quel degré de certitude il valide la relation prédicative. De ce fait «l'adverbe de modalité se caractérise à la fois par sa fonction inférentielle et par sa valeur épistémique ${ }^{19}$ " (Guimier 1988: 256). Bien entendu, chaque adverbe remplit les deux fonctions -inférentielle et épistémique- dans des proportions variables; par exemple, plainly a essentiellement une fonction inférentielle ${ }^{20}$, probably a essentiellement une fonction épistémique ${ }^{21}$, et, dans conceivably, les deux fonctions s'équilibrent plus ou moins.

J'ai relevé 74 adverbes de modalité dans mon corpus de travail (Morvan 2012 : 95)22. 6 ont une fonction épistémique prédominante et je n'en parlerai pas ici; 68 ont une fonction inférentielle prédominante, et un petit groupe d'adverbes (ou syntagmes prépositionnels à fonction adverbiale) est responsable de 59 occurrences sur 68 : easily, trivially, at once, obviously, clearly et of course ${ }^{23}$. Ces adverbes sont d'abord des marqueurs explicites du renforcement de la prise en charge de l'assertion par l'énonciateur et marquent donc une modalité assertive: l'énonciateur certifie la validation de la relation prédicative à l'intention du co-énonciateur (cf. \$3.2). En même temps, ces adverbes sont utilisés par l'énonciateur pour commenter la validation de la relation prédicative et ils marquent donc aussi une modalité appréciative. Notons que le commentaire, d'ordre qualitatif, a en général un fondement « objectif » : pour émettre son appréciation, l'énonciateur s'appuie sur des faits mathématiques qu'il n'explicite pas mais qu'il suppose connus du coénonciateur. On verra ci-dessous une classification des adverbes en trois groupes, selon la manière dont l'énonciateur fait appel (ou non) à ces faits mathématiques non explicités.

Premier cas : l'énonciateur déclare qu'il existe une preuve de l'assertion, mais il estime inutile de la fournir, parce qu'il la considère facile (easily, trivially) ou rapide (at once). Exemples :

(42) Uniqueness of factorization of ideals in D' easily yields $D^{\prime} a=D^{\prime} k$, proving (i) (S167)

(42) Indeed, one sees at once that $\mathrm{yG}_{\mathrm{s}} \mathrm{y}^{-1}$ leaves s' fixed, and that $\mathrm{y}^{-1} \mathrm{G}_{\mathrm{s}} \mathrm{y}$ leaves $\mathrm{s}$

fixed, whence the indicated equality follows. (L21)

Lorsque la proposition énoncée peut être démontrée rapidement et facilement, verify est utilisé de préférence à prove. Il n'est donc guère étonnant que les adverbes easily, trivially et at once soient fréquemment associés à verify. Exemple :

(44) It is trivially verified that $f$ is an isomorphism if and only if $f$ is bijective. (L10)

Notons en outre que, en utilisant trivially plutôt que easily dans (44), l'énonciateur indique que la démonstration envisagée est non seulement facile, mais tellement facile qu'elle en est sans intérêt ${ }^{24}$; la modalité marquée est plus appréciative avec trivially qu'avec easily.

Deuxième cas : l'énonciateur déclare qu'il considère l'assertion comme évidente et il estime inutile de se préoccuper de la preuve (obviously, clearly). Selon SimonVanderbergen \& Aijmer (2007: 166), « clearly and obviously are close together semantically and pragmatically ${ }^{25}$. It is difficult to pinpoint the differences between them». On peut le constater sur les deux exemples suivants : 
(45) Then $f$ is clearly a homomorphism. (L15)

(46) Then $N$ is obviously a subgroup of G. (L15)

Troisième cas : l'énonciateur déclare qu'il considère l'assertion comme évidente et il s'attend à ce que le co-énonciateur aussi l'accepte comme évidente (of course). «[This adverb] signal[s] conformity with [...] the speaker's and hearer's expectations" (SimonVanderbergen \& Aijmer 2007 : 38). Exemple :3

(47) Of course we used in the proof the fundamental theorem of algebra. (C143)

3 Of course peut aussi avoir une valeur concessive :

(48) Of course, $\delta(t-t))$ is not an ordinary function. However, says Dirac, let us formally operate with $\delta(t-t)$ as if it really were an ordinary function. (B244)

\subsection{Quelques remarques sur obviously, clearly, of course et naturally}

54 L'utilisation de obviously et clearly renforce une assertion dont l'énonciateur peut penser que l'énonciation sans modalisation ne serait pas suffisamment convaincante. Il s'agit en effet, dans la plupart des exemples recensés, de propositions intégrées à une partie à fonction intra-démonstrative, et qui ne sont justifiées par aucun raisonnement ou calcul. La présence de obviously ou clearly est censée, dans le cadre d'une relation intersubjective, encourager le co-énonciateur à valider quand même la relation prédicative. Par contre, of course est le plus souvent utilisé dans des parties à fonction métadiscursive. L'auteur annonce, commente ou complète des résultats démontrés par ailleurs. L'énonciateur a moins besoin de convaincre le co-énonciateur ; il se contente de lui indiquer, sans l'aider mais sans le contraindre, qu'il est « naturel » de valider l'assertion.

Par ailleurs, of course a une fonction discursive: "[it is] used to show that other people probably already know what you are saying is true, or expect to hear it » (Longman 2009 : 1207). "The meaning of of course includes the feature 'knowledge (that should be) shared by the adressee' " (Simon-Vanderbergen \& Aijmer 2007 : 176). Dans le cadre du discours écrit, où le coénonciateur ne peut répondre à l'énonciateur, l'utilisation de of course par l'énonciateur peut avoir pour but d'instaurer une connivence avec le co-énonciateur : on suggère à celui-ci que l'énoncé ne dit rien qui doive le surprendre, et donc qu'il lui faut accepter les assertions de l'énoncé. "Of course [...] presupposes 'as everyone knows' and can therefore be used as a rhetorical ploy" (Simon-Vanderbergen \& Aijmer 2007: 30) ${ }^{26}$. On peut effectivement trouver des exemples à l'appui de cette remarque :

(49) The volume $\mathrm{v}(\mathrm{X})$ of a subset $\mathrm{X} \subseteq \mathrm{Rn}$ is defined in the usual way: for precision we

take it to be value of the multiple integral $\int_{x} d x_{1} \ldots d x_{n}$ where $(\mathrm{x} 1, \ldots, \mathrm{xn})$ are coordinates. Of course the volume exists only when the integral does. (S141)

56 Ici, la deuxième proposition ne peut guère surprendre le lecteur : le volume étant défini par une intégrale, pour qu'il existe, il est nécessaire que l'intégrale existe. Autre exemple :

(50) The cases $n=1$ and $n=2$ are covered by Theorems 186 and 188. These theorems, of course, included a positive as well as a negative statement. (H161)

57 Si on se reporte au théorème 188 , on voit immédiatement que tel est bien le cas: THEOREM 188. A quadratic irrational is approximable to order 2 [affirmation] and to no higher order [négation]. (H158)

58 Simon-Vanderbergen \& Aijmer détectent une différence entre of course " [which] puts emphasis on the speaker's viewpoint that the hearer should [also] know 》 $(2007: 220)$ et naturally 
" [which] does not have the implication that the hearer knows or should know» (2007:236). Ainsi, dans l'exemple suivant :

(51) The irreducible polynomials relate to all polynomials in the same way that primes do to natural numbers. We naturally ask: Which polynomials are irreducible? The question clearly depends on the field $F$. (C136)

il est certes naturel de poser la question Which polynomials are irreducible? mais l'auteur aurait pu s'intéresser à d'autres questions. Donc la primauté donnée à celle-ci ne va pas de soi pour le lecteur et il lui paraitrait sans doute surprenant de lire : ?of course, we ask: Which polynomials are irreducible?

Remarquons pour finir que clearly ne semble pas instaurer une telle connivence entre l'énonciateur et le co-énonciateur: «In contrast with obviously, clearly has not developed an expectation sense» (Simon-Vanderbergen \& Aijmer 2007: 169). Cette différence entre clearly et obviously, impossible à mettre en évidence sur des énoncés brefs tels que (45) et (46), apparaitt par manipulation de l'énoncé (52) :

(52) Obviously, as regards the group-theoretic structure, a lattice of dimension $m$ is a free abelian group of rank $m$, so we can apply the terminology and theory of free abelian groups to lattices. (S135)

(52') Clearly, as regards the group-theoretic structure, a lattice of dimension $m$ is a free abelian group of rank $m$, so we can apply the terminology and theory of free abelian groups to lattices.

Dans (52'), on peut penser que l'énonciateur dit que la validation de la relation prédicative est évidente pour lui, mais qu'il ne présuppose pas qu'il en sera de même pour le co-énonciateur.

\subsection{Structures adjectivales extraposées}

61 J'ai relevé dans mon corpus de travail 45 occurrences de structures du type it is+adjectif+ that+complétive finie ou it is+adjectif+complétive non-finie (Morvan 2012: 113). Comme avec les adverbes, la modalité exprimée est généralement à la fois assertive et appréciative ${ }^{27}$. Les adjectifs les plus fréquents sont easy, clear et plain (24 occurrences au total). Exemples :

(53) If we define addition and multiplication by formulas (1) and (2), it is easy to see that $F$

$[x]$ is a commutative ring, and has no zero divisors. (C126)

(54) It is clear that the set of all such products is a subgroup of $G$ (the empty product is the unit element), and is the smallest subgroup of $G$ containing S. (L11)

Si la validité de la proposition the set of all such products is a subgroup of $G$ est évidente, on peut se demander pourquoi il est nécessaire d'asserter qu'elle l'est: " What use could it be to claim that a proposition is true if it is already accepted as true?» (Taranto 2008: 311). La réponse est que l'auteur anticipe l'existence, chez le lecteur, de doutes quant à la véracité de la proposition. Peut-être celui-ci n'a-t-il pas de connaissances mathématiques suffisantes pour la démontrer, ni même pour comprendre pourquoi elle est vraie. L'auteur ignore évidemment le niveau mathématique de ses lecteurs. Il doit donc à la fois les convaincre et les guider, en certifiant qu'il est possible de démontrer la proposition. Cette prise en charge de l'assertion est nécessaire; l'énonciateur doit harmoniser sa position avec celle du co-énonciateur avant de passer à la suite de son discours : " asserting clarity means that the proposition is clear enough to proceed as if it were true " (Taranto 2008: 322). Ce que l'énonciateur veut exprimer dans (54) pourrait être développé ainsi : It is clear for me that the set of all such products is a subgroup of G. I hope it is 
clear for you too. If not, I assure you that I can prove this proposition. In any case, we should agree that it is true before proceeding further.

Les adjectifs easy, trivial, clear, plain, obvious et natural ont la double caractéristique de pouvoir être inclus dans des structures adjectivales extraposées et de donner par adjonction de la terminaison -ly des adverbes de modalité, les deux marqueurs étant rencontrés dans mon corpus ${ }^{28}$. On peut donc s'interroger sur les raisons du choix effectué par l'auteur entre l'un ou l'autre de ces deux marqueurs de modalité. Lorsque l'adverbe est placé en début de phrase, il parait difficile de déceler une différence sémantique ou énonciative induite par le passage de l'une à l'autre des deux structures :

(55) Clearly $\delta(\alpha+\beta)-\delta(\alpha)+\delta(\beta)($ (s153)

$\left(55^{\prime}\right)$ It is clear that $\delta(\alpha+\beta)-\delta(\alpha)+\delta(\beta)$

Par contre, dans les énoncés où l'adverbe n'est pas en début de phrase, son remplacement par une structure extraposée permet à l'énonciateur de mettre en relief l'expression de son jugement, qui prend de ce fait un caractère plus autoritaire.

(56) One verifies easily that the map $\mathrm{x} \mapsto \mathrm{x}^{\mathrm{n}} \ldots$ from $\mathrm{G}$ into itself is a homomorphism.

(L11)

(56') It is easy to verify that the map $\mathrm{x} \mapsto \mathrm{x}^{\mathrm{n}}$ from $\mathrm{G}$ into itself is a homomorphism.

Dans (56'), comme le permet l'opérateur à valeur de visée to, l'énonciateur indique par avance que la vérification, si elle est faite, se révélera facile. A contrario, l'utilisation de l'adverbe dans (56) invite le co-énonciateur à accepter l'assertion en même temps qu'il la découvre.

\section{Conclusion}

Bien entendu, le discours mathématique pédagogique en anglais ne pouvait échapper à la fréquente utilisation des auxiliaires modaux dans cette langue. Un texte mathématique à visée pédagogique ne peut se résumer à une suite de calculs, d'abord parce que des raisonnements doivent ordonner et structurer ces calculs, ensuite parce que des explications et des commentaires doivent les accompagner. Pour des raisons pratiques évidentes, l'auteur ne peut pas détailler tous les calculs et raisonnements; pourtant il doit quand même convaincre ses lecteurs. En conséquence, l'énonciateur est amené fréquemment à marquer explicitement sa prise en charge des énoncés.

Certaines caractéristiques du discours mathématique, telles que l'effacement de l'énonciateur et l'établissement de résultats incontestables, ont permis d'expliquer la surreprésentation de shall. La présence de nombreux énoncés posant des hypothèses ou donnant des conclusions a permis de mettre en évidence et de justifier l'utilisation préférentielle de may dans le premier cas et de can dans le second cas. Enfin il a été possible de classer en trois groupes les adverbes de modalité à fonction inférentielle dominante les plus fréquents, puis, à l'intérieur de deux de ces trois groupes, de présenter quelques critères et quelques conséquences des choix effectués.

Le discours mathématique a été jusqu'ici peu étudié par les linguistes et on pourrait se pencher sur d'autres phénomènes, comme par exemple -en restant dans le prolongement du présent article- l'utilisation de la forme passive et en particulier les structures du type it is to/can/may be observed/proved that. Bien entendu, il conviendrait aussi d'élargir l'étude à d'autres formes de textes mathématiques : articles de recherche et ouvrages scolaires. 


\section{BIBLIOGRAPHIE}

Braun, Martin. 1993. Differential Equations and their Applications. New York : Springer Verlag. $233-263^{29}$.

Childs, Lindsay. 1979. A Concrete Introduction to Higher Algebra. New York : Springer-Verlag. 125-156.

Grove, Wendell. 1966. Brief Numerical Methods. Englewood Cliffs : Prentice-Hall. 78-109.

Hardy, G.H. et E.M. Wright. 1979. An Introduction to the Theory of Numbers, fifth edition. Oxford :

Clarendon Press. 154-177.

Lang, Serge. 1971. Algebra. Reading (Mass.) : Addison-Wesley. 5-25.

Stewart, Ian et David Tall. 1979. Algebraic Number Theory. London : Chapman \& Hall. 135-175.

Bouscaren, Janine et Jean Chuquet. 1987. Grammaire et textes anglais. Guide pour l'analyse linguistique . Gap : Ophrys.

Courtès, Joseph. 1991. Analyse sémiotique du discours. De l'énoncé à l'énonciation. Paris : Hachette.

Gilbert, Eric. 1987. May, Must, Can et les opérations énonciatives. Cahiers de recherche, tome 3. Gap : Ophrys.

Gilbert, Eric. 1993. « La théorie des opérations énonciatives d'Antoine Culioli, 'La modalité' », in Cotte, P. et al. (dir.) Les théories de la grammaire anglaise en France. Paris : Hachette. 63-96.

Gilbert, Eric. 2001. « Vers une analyse unitaire des modalités. » in Bouscaren, J. (dir.) Cahiers de Recherche, tome 8. Gap : Ophrys. 23-99.

Gresset, Stéphane. 2001. «Can/may et might/could ou l'interchangeabilité à l'épreuve des textes. » in Bouscaren, J. (dir.) Cahiers de Recherche, tome 8. Gap : Ophrys. 177-222.

Guimier, Claude. 1988. Syntaxe de l'adverbe anglais. Lille : Presses Universitaires de Lille.

Hoye, Leo. 1997. Adverbs and Modality in English. London : Longman.

Larreya, Paul. 1984. Le possible et le nécessaire. Modalités et auxiliaires modaux en anglais britannique. Paris : Nathan.

Le Querler, Nicole. 1996. Typologie des modalités. Caen : Presses Universitaires de Caen.

Longman Dictionary of Contemporary English. 2009. Edinburgh : Pearson.

Morvan, Eric. 2012. Modalisation dans le discours mathématique écrit en anglais. Mémoire de master : Université de Poitiers.

Nordon, Didier. 1999. Deux et deux font-ils quatre? Sur la fragilité des mathématiques. Paris : Belin.

Petit, Michel. 1993. Analyse contrastive de la relation discursive et inter-énonciative dans un corpus d'ouvrages mathématiques en français et en anglais. Thèse : Université de Paris VII.

Simon-Vandenbergen, Anne-Marie et Karin Aijmer. 2007. The Semantic Field of Modal Certainty. A Corpus-Based Study of English Adverbs. Berlin, New York : Mouton-De Gruyter.

Sionis, Claude. 2002. «Quelques spécificités de la modalisation dans le discours scientifique ». ASp , $\mathrm{n}^{\circ} 35-36.39-59$ 
Taranto, Gina. 2008. « Discourse adjectives » in McNally, C. et Kennedy, C. (eds.) Adjectives and Adverbs : Syntax, Semantics, and Discourse. Oxford : Oxford University Press. 305-327.

Varttala, Teppo. 2001. Hedging in Scientifically Oriented Discourse. Exploring Variation According to Discipline and Intended Audience. Doctoral Dissertation : Université de Tampere, consulté le 15/10/2012. <http://acta.uta.fi/pdf/951-44-5195-3.pdf>

Vold, Eva. 2008. Modalité épistémique et discours scientifique. Thèse : Université de Bergen. Webster's New Encyclopedic Dictionary. 1994. Cologne : Könemann.

\section{NOTES}

1. Le sujet énonciateur est un concept théorique linguistique. Il prend en charge le discours produit par un locuteur ou le texte produit par un auteur. Le locuteur ou l'auteur appartiennent à la réalité extra-linguistique.

2. Chaque exemple est repéré par une lettre désignant l'ouvrage d'où il provient suivie du numéro de la page d'origine. Les lettres sont B pour le livre de Braun, C pour le livre de Childs, $G$ pour le livre de Grove, $\mathrm{H}$ pour le livre de Hardy \& Wright, L pour le livre de Lang et S pour le livre de Stewart \& Tall.

3. Les résultats sont en général précédés des titres Theorem, Proposition, Lemma ou Corollary.

4. Rappelons brièvement cette classification (cf. Bouscaren \& Chuquet 1987, Gilbert 1993) : dans la modalité de type 1 , dite assertive, l'énonciateur reste «neutre » quant à la validation de la relation prédicative ; dans la modalité de type 2, dite épistémique, il évalue quantitativement les chances de validation de la relation prédicative ; dans la modalité de type 3 , dite appréciative, il porte un jugement qualitatif sur la relation prédicative dans son ensemble ; dans la modalité de type 4 , dite radicale, il émet un jugement sur la capacité ou la volonté du sujet à réaliser le procès exprimé dans la relation prédicative (modalité dynamique) ou sur les contraintes qui s'exercent sur le sujet (modalité déontique).

5. Il n'est pas possible de donner un décompte précis car certaines parties de texte ne relèvent ni de la fonction intra-démonstrative, ni de la fonction métadiscursive, et, inversement, dans d'autres parties, ces deux fonctions sont développées simultanément.

6. On dit que $P$ est une condition nécessaire de $Q$ ou que $Q$ est une condition suffisante de $P$.

7. Compte tenu de l'existence de cette relation d'implication de $Q$ vers $P$, « on n'est pas en présence d'un nécessaire «absolu » [...] mais d'un nécessaire « relatif », qui n'est tel que par la mise en rapport de deux relations prédicatives» (Gilbert 1987 : 193). Il s'agit ici de la modalité implicative étudiée par Larreya (1984).

8. Les citations entre guillemets sont d'A. Culioli.

9. Ainsi, dans I will always love you!, du fait de la valeur de volition de wiLl, l'énonciateur exprime une intention. Dans I shall always love you!, l'énonciateur exprime la permanence de son sentiment, auquel sa volonté est soumise.

10. Comme le signale par ailleurs E. Gilbert (2001: 86), cette analyse ne fait pas l'unanimité des grammairiens.

11. 53 occurrences de we shall contre 18 occurrences de we will (Morvan 2012: 78). Voir exemples (17), (18) et (19).

12. L'ouvrage de W.Grove est un manuel de mathématiques appliquées.

13. Cette remarque reste valable lorsque CAN et MAY ont une interprétation déontique : «la différence entre MAY et CAN dans l'expression de la permission réside bien dans la différence de statut accordée à l'altérité [...] : celle-ci est explicitement prise en compte avec MAY alors qu'avec CAN rien n'est dit » (Gresset $2001: 184)$. 
14. 9 occurrences (Morvan $2012: 44$ ).

15. 20 occurrences avec CAN, 2 avec MAY (Morvan $2012: 45$ ).

16. Restent deux occurrences qui correspondent à une définition et à une question, où le problème de la démonstration ne se pose donc pas.

17. Bien que semblables, les transformations mathématiques exposées sont de complexité croissante quand on va de (39) à (40) à (41). La nécessité d'encourager le lecteur croît parallèlement.

18. Au moins deux : considérer $\mathrm{F}[\mathrm{x}, \mathrm{y}, \mathrm{z}]$ comme polynômes en $\mathrm{y}$ avec des coefficients polynomiaux en $\mathrm{x}$ et $\mathrm{z}$, ou considérer $\mathrm{F}[\mathrm{x}, \mathrm{y}, \mathrm{z}]$ comme polynômes en $\mathrm{x}$ avec des coefficients polynomiaux en $\mathrm{y}$ et $\mathrm{z}$.

19. Le fait que Guimier parle de "fonction » inférentielle et de "valeur » épistémique est sans doute dû au fait qu'il caractérise en premier lieu les adverbes de modalité comme des adverbes d'inférence (1988: 253) ; mais il utilise aussi le terme de « valeur » inférentielle (1988:256).

20. Voir exemple (13).

21. Voir exemple (16).

22. En l'absence d'un consensus des linguistes sur la définition d'un adverbe de modalité, il n'est pas facile de décider quels adverbes doivent être considérés comme tels. Je me suis appuyé sur Hoye (1997), Guimier (1998) et Simon-Vanderbergen \& Aijmer (2007). Un exemple : je n'ai pas retenu simply ou merely parce qu'ils peuvent être, soit adverbes modificateurs de mot, soit adverbes modificateurs de phrase. A contrario j'ai retenu plainly bien qu'une phrase comme: We

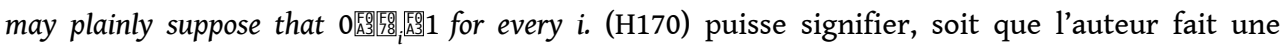
hypothèse évidente et simple, soit que le choix de faire cette hypothèse est un choix évident et simple. Enfin, je n'ai pas pris en compte les adverbes marquant une modalité purement appréciative, tels happily, sadly, fortunately, qui ne relèvent pas de la définition choisie par Guimier; de tels adverbes se rencontrent de toute façon fort peu dans mon corpus. En voici toutefois un exemple : Otherwise, it will take an awfully long time to do this problem. (B263).

23. Les autres adverbes relevés sont immediately, plainly, admittedly et naturally (Morvan 2012: 96). 24. «Trivial [...] not serious, important or valuable» (Longman 2009: 1888). «2: of little worth or importance » (Webster 1994: 1112).

25. Certaines des définitions données par le Longman Dictionary of Contemporary English (2009) sont effectivement très proches. Pour obviously: « used to mean that a fact can easily be noticed or understood » (1202). Pour clearly: «1-without any doubt [...] 2- in a way that is easy to see, hear or understand » (298).

26. obviously peut aussi, comme of course, véhiculer le message selon lequel l'énoncé à venir ne surprendra pas le co-énonciateur, comme le montre l'énoncé suivant: Note that in order to use equation (7.14) to compute $y_{n+1}$, we must already know $y_{n+1}^{\prime}$, which of course requires that we know $y_{n+1}$. Obviously we must have a way to find $y_{n+1}{ }^{\circ}$ (G102)

27. Il y a quelques exemples de modalité purement appréciatives : cf. exemple (15).

28. 25 occurrences de structures adjectivales et 45 occurrences d'adverbes (Morvan 2012: 96, 113-115).

29. Les pages mentionnées sont celles d'où ont été tirés tous les exemples d'énoncés modalisés. 


\section{RÉSUMÉS}

Cet article présente une étude quantitative et qualitative des phénomènes de modalisation dans le discours mathématique en anglais, en s'appuyant sur un corpus extrait d'ouvrages pédagogiques de niveau universitaire. Les marqueurs de modalisation pris en compte sont les auxiliaires modaux, ainsi que certains adverbes et structures adjectivales extraposées. L'étude s'appuie sur la théorie des opérations énonciatives. Après avoir examiné quelques caractéristiques du discours mathématique, nous tentons de montrer pourquoi et comment ces caractéristiques influent sur les règles généralement constatées d'utilisation des modalisateurs en langue anglaise. Le contexte du discours mathématique permet en outre d'affiner ou d'enrichir certaines interprétations des modalisateurs étudiés.

This article offers a quantitative and qualitative study of modalization in mathematical discourse in English. It is based on a corpus made up of extracts from university textbooks for undergraduate and graduate levels. The modalization markers taken into account are the modal auxiliaries, along with some adverbs and extraposed adjectival structures. The approach is that of the theory of enunciative operations. After examining a few features of mathematical speech we try to show why and how these features alter the commonly accepted rules of modalization in the English language. The context of mathematical discourse will be used to refine and expand some interpretations of the modalization markers studied.

\section{INDEX}

Mots-clés : discours mathématique, auxiliaires modaux, adverbes de modalité

Keywords : mathematical discourse, modal auxiliaries, modality adverbs

\section{AUTEUR}

\section{ERIC MORVAN}

Université de Poitiers, Laboratoire FORELL 